\title{
Atypical post-surgical guillain-barré with a fluctuating thoracic sensory level
}

\begin{abstract}
Guillain-Barré Syndrome (GBS) is an acute demyelinating polyneuropathy. It is usually preceded by an infection. Typically, GBS presents with progressive symmetric weakness with diminished reflexes. Autonomic involvement is also frequent. Here, we report a case of atypical GBS presentation in a 57-year-old man following lumbar stenosis surgery. The patient presented with a fluctuating thoracic sensory level and preserved reflexes until well into his motor symptoms. Atypical presentations after surgery and with spinal sensory levels are uncommon but require acute recognition and treatment.
\end{abstract}

Keywords: guillain-barré syndrome, lumbar stenosis, orthopedic surgery, neurology, post-surgical, demyelinating, fluctuating, progressive, emergency, consequences, involvement, post-operatively, fluctuated; asymmetric, polyneuropathy, demyelinating
Volume 4 Issue 2 - 2017

\author{
Melissa MJ Chua MA,' Jami H Johnsen MD, ${ }^{2}$ \\ Chadi A Tannoury MD,' Anna D Hohler MD,' \\ 'Boston University School of Medicine, USA \\ ${ }^{2}$ Boston Medical Center, USA
}

Correspondence: Melissa MJ Chua, Boston University School of Medicine, MA, 57 E Concord St Boston, USA, 021 I8 Tel 617. 935-4032, Email mel98791@bu.edu

Received: October 01, 2016 | Published: March 07, 2017

\section{Introduction}

Guillain-Barré Syndrome (GBS) is an acute demyelinating polyneuropathy, mediated by autoimmune attack on peripheral nerves, and is usually preceded by an infection. Patients generally present with progressive symmetric weakness and decreased or absent reflexes. The majority of patients also present with facial weakness, distal paresthesia, and autonomic dysfunction. GBS is diagnosed through lumbar puncture and neurophysiology studies. Elevated cerebrospinal fluid (CSF) protein and decreased or blocked nerve conduction are diagnostic of GBS. GBS following spinal surgery is uncommon and not well-documented. Occasionally the symptoms of GBS can be misattributed to post-surgical complications. Here, we report a case of atypical GBS presentation following lumbar spinal stenosis surgery.

\section{Case presentation}

A 57year-old male had lumbar stenosis and surgical correction with an anterior and posterior lumbar inter body fusion of L3-S1. After the surgery, his pain and strength improved and he was able to ambulate without his walker. Hethen presented to the emergency department nine days post-operatively with left leg paresthesias and weakness. His left leg was affected more significantly than his right, and he had difficulties with ambulation. Physical examination revealed weakness to $3 / 4$ in the left leg and $4 / 5$ in the right leg both proximally and distally. Reflexes were preserved in the arms and legs. Sensation was decreased in both legs to pin prick and he had a T12 sensory level. Over the next few days, he had further progressive leg weakness and then dropped reflexes. His workup included spine imaging which did not reveal any acute changes (Figure 1). Nerve conduction studies showed prolonged distal motor latencies, conduction blocks, as a well as sural sparing, consistent with a demyelinating polyneuropathy, specifically GBS. He was initiated on intravenous immunoglobulin (IVIG) therapy at a dose of $400 \mathrm{mg} / \mathrm{kg}$ over five days. A lumbar puncture to assay CSF was not performed due to his post-lumbar surgical status and the confirmatory nature of the EMG. His sensory symptoms in his trunk fluctuated. There was also progressive cranial nerve involvement, with left facial weakness. He developed autonomic issues including urinary retention and atrial fibrillation, and was eventually incubated due to impending respiratory failure. The patient underwent a second cycle of a five day IVIG therapy course. Both weakness and sensation eventually improved. On first examination in the Emergency Department, GBS was not suspected as spinal level sensory involvement and asymmetric weakness with preserved reflexes is typically not characteristic of GBS. Additionally, because of his recent surgical history, his symptoms were thought to arise from possible post-surgical complications. The patient also did not have any antecedent infections. With the rapid progression of symptoms and then diminishing reflexes, GBS was then considered. Two months post-discharge, the patient began ambulating. His overall weakness has improved and has regained strength in his extremities. His only residual symptom is foot drop on the left. He is otherwise back to his baseline and had had return of his former excellent quality of life.
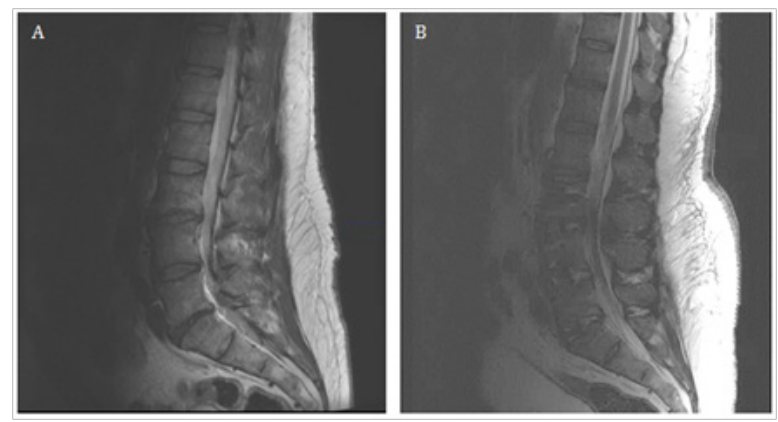

Figure I Magnetic resonance imaging of the lumbar spine.

A. Pre-operative sagittal T2-weighted image showing severe moderate lumbar stenosis at L3-L4, L4-5 and severe lumbar stenosis at L5-SI levels.

B. Post-operative anterior and posterior lumbar interbody fusion of L3-SI sagittal T2-weighted image showing reversal of cord compression. 


\section{Discussion}

This is an uncommon case of GBS after lumbar spinal surgery with sensory involvement. There has only been one other reported case of truncal sensory level as an early GBS manifestation. ${ }^{1}$ There is scarce literature on GBS post-surgery. Gensicke et al. reported a 13.1 times higher risk of GBS within the first 6 weeks post-surgery compared to the general population. ${ }^{2}$ Additionally, Jung et al. reported a case of lumbar stenosis which was later diagnosed as GBS. ${ }^{3}$ This literature and our case suggests that atypical GBS with sensory involvement can manifest following lumbar spinal stenosis surgery. GBS should be in the differential when a patient presents with weakness post-surgery as it can lead to serious consequences when missed. Current treatment options for GBS include IVIG and plasma exchange, with both being equally efficacious. ${ }^{4}$ IVIG is generally recommended for patients who are unable to ambulate and are able to begin treatment two to four weeks from the onset of symptoms. Plasma exchange is recommended for patients who are unable to ambulate and are able to begin treatment within four weeks of the onset of symptoms, or for patients who are ambulatory and able to begin treatment within two weeks of the onset of symptoms. If a patient responds poorly to treatment, another course of the same treatment should be administered after observations. ${ }^{5}$

\section{Ethical standards}

Permission to publish information related to the case has been approved by the patient and his/her identity is protected.

\section{Acknowledgements}

None.

\section{Conflict of interest}

The author declares no conflict of interest.

\section{References}

1. Alfahad TB, Kelly JJ. Pure T7 sensory level as an isolated manifestation of guillain-barré syndrome. J Clin Neuromuscul Dis. 2014;15(3):102-104.

2. Gensicke H, Datta AN, Dill P, et al. Increased incidence of guillain-barré syndrome after surgery. Eur J Neurol. 2012;19(9):1239-1244.

3. Jung DY, Cho KT, Lee SC. Atypical guillain-barré syndrome misdiagnosed as lumbar spinal stenosis. J Korean Neurosurg Soc J Korean Neurosurg Soc. $2013 ; 53(4): 245$.

4. Hughes RA, Wiidicks EF, Barohn R, et al. Practice parameter: immunotherapy for guillain-barré syndrome: report of the quality standards subcommittee of the american academy of neurology. Neurology. 2003;61(6):736-740

5. Winer JB. when the Guillain-Barre patient fails to respond to treatment. Pract Neurol. 2009;9(4):227-230. 